\title{
Size dependence of core and valence binding energies in Pd nanoparticles: Interplay of quantum confinement and coordination reduction
}

\author{
I. Aruna, ${ }^{1, b)}$ B. R. Mehta, ${ }^{1, a)}$ L. K. Malhotra, ${ }^{1}$ and S. M. Shivaprasad ${ }^{2, c)}$ \\ ${ }^{1}$ Thin Film Laboratory, Department of Physics, Indian Institute of Technology Delhi, \\ New Delhi 110 016, India \\ ${ }^{2}$ Surface Physics and Nanostructures Group, National Physical Laboratory, New Delhi 110 012, India
}

(Received 26 March 2008; accepted 26 June 2008; published online 23 September 2008)

\begin{abstract}
An analysis is given of the electronic structure of Pd nanoparticles synthesized by inert gas evaporation technique. A study of the effect of size on various core and valence electrons in Pd nanoparticles reveals a varied dependence of binding energy of electrons in different electronic levels. The shift in the Pd x-ray photoelectron spectroscopy $4 d$ valence band centroid is more than the core level shift. The results of the present study provide a direct evidence of interplay of quantum confinement (a size effect) and coordination reduction (a surface effect). (C) 2008 American Institute of Physics. [DOI: 10.1063/1.2973682]
\end{abstract}

\section{INTRODUCTION}

Size dependent changes in the optical, structural, and electrical properties are inherently related to the basic changes in the electronic structure of materials at nanodimensions. X-ray photoelectron spectroscopy (XPS) can provide important information on the influence of nanoparticle size on electronic properties. The common observation in the XPS studies of metal nanoparticles reported in the literature $(\mathrm{Au}, \mathrm{Ag}, \mathrm{Pd}, \mathrm{Pt}, \mathrm{Ni}, \mathrm{Cu}$, and $\mathrm{Co}$ ) is the positive core level shift with respect to the bulk values. ${ }^{1-9}$ In a few studies, shifts in the binding energy of valence band with respect to Fermi level and narrowing of valence band spectra have also been reported. ${ }^{3,7,8}$ Although considerable efforts have been focused on elucidating the physical origin of the observed size dependent changes, the interpretation of the observed behavior is still unclear. The core and valence level shifts have been interpreted in terms of the initial and final state effects. The former is related to the electronic structure of nanoparticles and the state before photoemission, while the latter is related to the positive charge left on the surface of the nanoparticles during photoemission process, the relaxation, and screening of core holes. Mason ${ }^{1}$ reviewed the shifts in the binding energy of core electrons ( $\mathrm{Au}, \mathrm{Ag}, \mathrm{Pd}, \mathrm{Pt}$, $\mathrm{Cu}$, and $\mathrm{Ni}$ deposited on amorphous carbon) and changes in the valence band (Pt and Pd) observed in XPS studies on metallic clusters reported in literature and argued that changes in the electron configuration of the atoms in smaller clusters were primarily responsible for the shift and that final state processes were relatively more important only for the reactive substrates. Wertheim et al., ${ }^{3}$ on the other hand, assigned the core level shift in $\mathrm{Ag}$ and $\mathrm{Pd}$ clusters grown on

\footnotetext{
${ }^{b}$ Present address: Institute of Nano Structures and Technology, Faculty of Engineering, University of Duisburg-Essen, Bismarckstrasse 81, 47057 Duisburg, Germany.

a) Author to whom correspondence should be addressed. Electronic mail: brmehta@physics.iitd.ernet.in.

${ }^{c}$ Present address: Chemistry and Physics of Materials Unit, Jawharlal Nehru Centre for Advanced Scientific Research, Jakkur, Bangalore 560064, India.
}

amorphous carbon due to final state screening effects. Wu et $a l .{ }^{6}$ studied the changes in the binding energy of $\mathrm{Cu} 2 p_{3 / 2}$ in $\mathrm{Cu}$ (thickness $<2 \mathrm{~nm}$ ) deposited on $\mathrm{Al}_{2} \mathrm{O}_{3}$ grown on $\operatorname{Re}(0001)$ substrate and argued that initial state contributions were small and significant for very small clusters. Zhang and Sham $^{8}$ studied the electronic properties of the thiol-capped Au nanoparticles $(1<R<5 \mathrm{~nm})$ using XPS and argued that the modifications in the binding energy and the full width at half maximum (FWHM) of the Au $4 f$ core levels and the valence $5 d$ band are mainly due the initial state effects. Whereas, Richter et al. ${ }^{9}$ studied the Co clusters $(R<1 \mathrm{~nm})$ deposited on $\mathrm{Al}_{2} \mathrm{O}_{3}$ grown on NiAl (110) by Auger parameter analysis along with theoretical modeling and argued that the initial and final state contributions to the core level shifts obtained were of comparable magnitude. Thus there is a substantial disagreement over the assignment of these shifts with initial or final state effects. In addition, in all the reported studies, only the most intense core level and the valence band have been investigated. Attempts to investigate and understand the effect of nanoparticle size on all the electronic levels of any metallic system are seldom encountered in literature. It may be mentioned here that in most of the reported studies on size dependence of the electronic properties of metal ( $\mathrm{Au}, \mathrm{Ag}, \mathrm{Pd}, \mathrm{Pt}, \mathrm{Ni}, \mathrm{Cu}$, and $\mathrm{Co}$ ) at nanodimensions, the samples were prepared by evaporating a small quantity of metal onto the substrate. ${ }^{1-6,9}$ The isolated metal islands normally have a rather broad and often not well characterized size distribution. The electronic properties were studied as a function of coverage, which is approximately mentioned as the particle size. The increase in particle size was achieved by increasing the deposition time and hence the coverage area. This can result in isolated islands at small coverage but overlapping particles at higher coverage. In a few studies (e.g., on Ag) the size of as-deposited islands was reduced by ion bombardment. ${ }^{7}$ Ion bombardment may modify the structural and electronic properties of the particles. In another study, surface-passivated metal $\mathrm{(Au})$ nanoparticles were prepared by chemical routes. ${ }^{8}$ In case of chemically prepared 
nanoparticles, the capping layer may significantly modify the electronic properties of the metal nanoparticles. In the present study, on the other hand, a continuous layer of Pd nanoparticles with a narrow size distribution has been deposited by the simple inert gas evaporation technique, wherein the particle size is varied by varying the deposition pressure. In addition to the importance of Pd as catalytic agent, the advantage of Pd in this investigation is the stability toward oxidation. The Pd nanoparticles in the present study have been deposited on doped Si substrates to minimize charging effects. In addition, the sample comprises of a continuous layer of Pd nanoparticles, and thus charging effects are expected to be minimal. To compensate for any possible charging effects, $\mathrm{C}(1 s)$ peak due to adsorbed carbon on the samples was used for internal calibration of peak positions. We report a detailed investigation of the size dependent modifications in the electronic properties of Pd nanoparticles, revealing a novel interplay between the quantum size effect and coordination reduction resulting in energy shifts by varying the amount in the various core and valence electron peaks.

\section{EXPERIMENTAL}

Pd nanoparticle layers have been deposited using inert gas evaporation technique. ${ }^{10}$ Prior to deposition, the growth chamber has been evacuated to $1 \times 10^{-6}$ Torr, and repeated argon purging was carried out to minimize the oxygen concentration in the growth chamber. High deposition pressure $\left(10^{-4}-10^{-3}\right.$ Torr of Ar), low substrate temperature $\left(T_{s}\right.$ $=30^{\circ} \mathrm{C}$ ), and high flow rate of argon $(\mathrm{FR}=20 \mathrm{SCCM})$ (SCCM denotes cubic centimeter per minute at STP) resulted in the formation of Pd nanoparticles. In the present study, the electronic properties of $\mathrm{Pd}$ layers deposited at 1.7 $\times 10^{-3}$ Torr and $3.9 \times 10^{-4}$ Torr pressures of argon (samples N1 and N2, respectively) and those deposited at 1 $\times 10^{-6}$ Torr vacuum (sample N3) have been investigated in detail. Transmission electron microscope (TEM) (Philips CM12 twin microscope, $120 \mathrm{keV}$, LaB6 cathode), glancing angle x-ray diffractometer (GAXRD) (Geigerflex-D/maxRB-RU200, Rigaku), and X-ray photoelectron spectrometer (XPS) (Perkin Elmer-1257 using a $M g K \alpha$ radiation of $E$ $=1253.6 \mathrm{eV}$ and a hemispherical section analyzer with 25 $\mathrm{meV}$ resolution) were used to characterize $\mathrm{Pd}$ nanoparticle layers. For TEM measurements the samples were prepared by depositing Pd directly onto the carbon coated TEM grids (300 mesh). Glass substrate was used for x-ray diffraction (XRD) measurements, whereas for XPS studies, doped Si wafer was used as substrate. It is important to note here that in Pd XPS valence band, the $4 d$ density of states has a sharp peak just below the Fermi level $\left(E_{F}\right)$; consequently, the fitting of the Fermi cutoff into the valence band requires an explicit representation of the density of states even for the bulk metal. Furthermore, the density of states of the bulk Pd, which is known, will also be modified for the nanoparticles with results that are difficult to be estimated by XPS. The complicated density of states also inhibits an analysis of the core level spectrum of bulk Pd. Because the $d$ band ends just above the $E_{F}$, the core electron spectrum for bulk Pd deviates

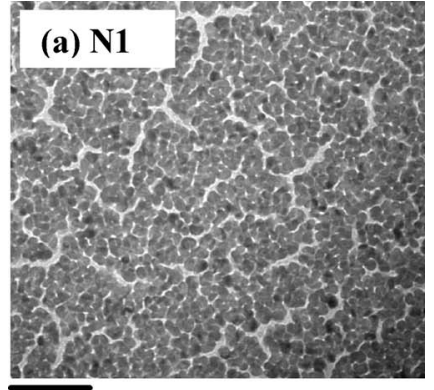

$25 \mathrm{~nm}$

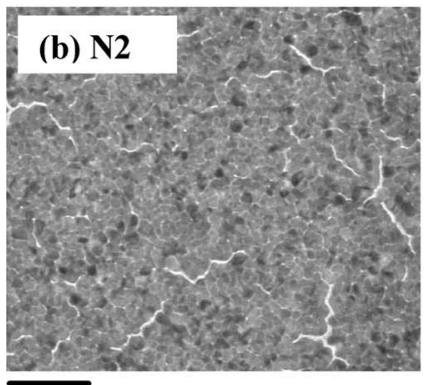

$25 \mathrm{~nm}$

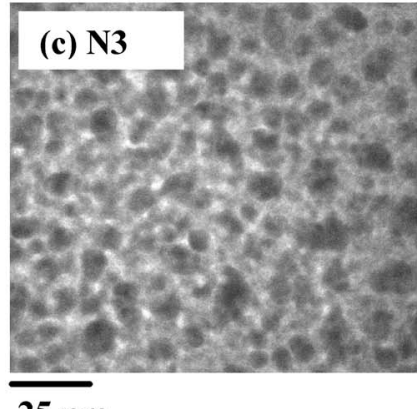

$25 \mathrm{~nm}$

FIG. 1. TEM micrographs of Pd samples (a) N1, (b) N2, and (c) N3.

strongly from the power-law line shape observed in simple metal. ${ }^{11}$ Thus in the present study, for all particle sizes, the positions of maxima of the Pd core electron levels and the centroid of the valence $4 d$ band have been used as a measure of the binding energy. It may also be noted that the FWHM of the $M g K \alpha$ line used for XPS analysis is $0.6 \mathrm{eV}$, and thus the energy resolution is expected to be of the order of 0.06 $\mathrm{eV}\left(10 \%\right.$ of the FWHM). ${ }^{12}$

\section{RESULTS AND DISCUSSION}

The TEM micrographs of the Pd layers N1, N2, and N3 shown in Fig. 1 reveal the nanoparticle nature and the estimated average particle sizes in these layers are 6, 11, and 20 $\mathrm{nm}$, respectively. TEM results reveal a decrease in the particle size with an increase in the deposition pressure. X-ray diffractograms of the Pd nanoparticle layers, Fig. 2(a), show predominant (111) peak along with (200), (220), and (311) peaks of fcc lattice. ${ }^{13}$ It is worthwhile to note a small shift in the position of diffraction peaks toward lower $2 \theta$ values with decrease in the particle size, revealing a size dependent lattice contraction. The estimated lattice parameter values in samples N3, N2, and N1 are 3.906, 3.900, and $3.894 \AA$, respectively. A similar lattice contraction has been reported 

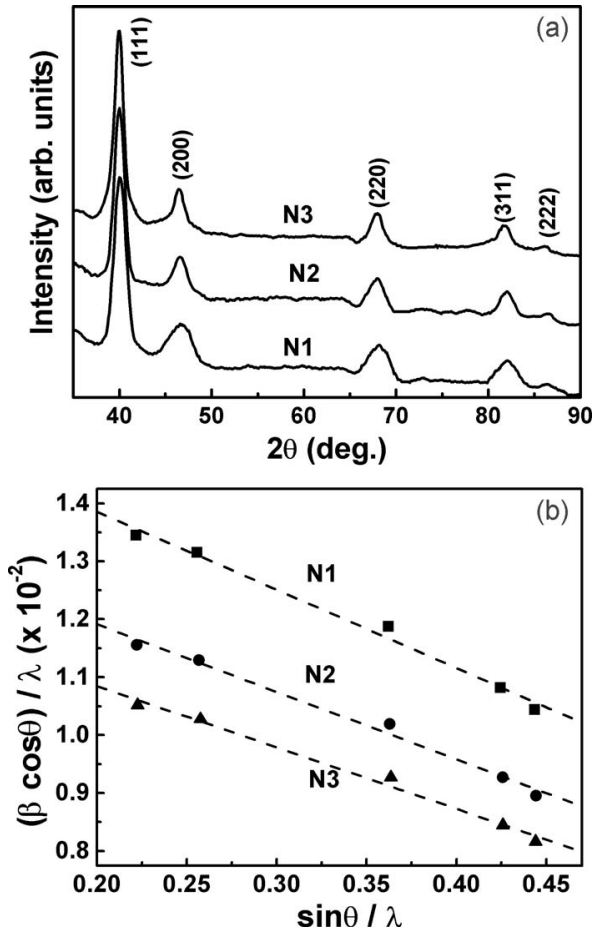

FIG. 2. (a) X-ray diffractograms and (b) $(\beta \sin \theta) / \lambda$ vs $(\sin \theta) / \lambda$ plots of Pd samples N1, N2, and N3.

in $\mathrm{Au}, \mathrm{Ni}, \mathrm{Sn}$, and $\mathrm{Bi}$ metal nanoparticles with decrease in size. ${ }^{8,14,15}$ As compared to bulk, Au nanoparticles undergo a lattice contraction of $0.7 \%$ on reduction in size at $4 \mathrm{~nm}$ and $1.4 \%$ at a size of $1.6 \mathrm{~nm}^{8}{ }^{8}$ In $5 \mathrm{~nm} \mathrm{Ni}$ particles, a lattice contraction of $2.4 \%$ has been observed. ${ }^{14}$ The shift in the XRD peaks has been attributed to the lattice distortion accompanied with the strain in the lattice in many metal nanoparticles and has been attributed to the surface bond that contracts with coordination reduction in surface atoms and the surface to volume ratio that increases with decreasing particle size. ${ }^{15}$ The effective strain $\eta$ in the lattice can be obtained using the Williamson Hall equation ${ }^{16}$ from the slope of $(\beta \cos \theta) / \lambda$ versus $(\sin \theta) / \lambda$ plots [Fig. 2(b)]. Here $\beta$ is the measured FWHM in radians, $\theta$ is the Bragg angle for the peak, and $\lambda$ is the $x$-ray wavelength. A linear variation with negative slope of $(\beta \cos \theta) / \lambda$ versus $(\sin \theta) / \lambda$ plots has been observed for Pd nanoparticle samples N1, N2, and N3. The magnitude of slope and hence the lattice strain is maximum for sample N1 and decreases with increase in particle size. The lattice strain is observed to change the chemical bonding between the metal atoms, which, in turn, is observed to induce modification in the electronic structure, ${ }^{9}$ as will be discussed later in the text.

XPS survey spectra of sputter cleaned Pd nanoparticle samples N1, N2, and N3 are shown in Fig. 3. The XPS spectra from the surface of the samples show only Pd XPS and Auger transitions, in addition to the peaks corresponding to the $C 1 s$. The $C 1 s$ peak disappears on sputter cleaning of the samples. The absence of substrate peaks in the XPS spectra of the samples clearly indicates that the Pd over layer fully covers the underlying $\mathrm{Si}$ substrate, simplifying the interpretation of the Pd XPS results. XPS spectra of Pd $3 d$ core electrons are shown in Fig. 4(a). It is interesting to note

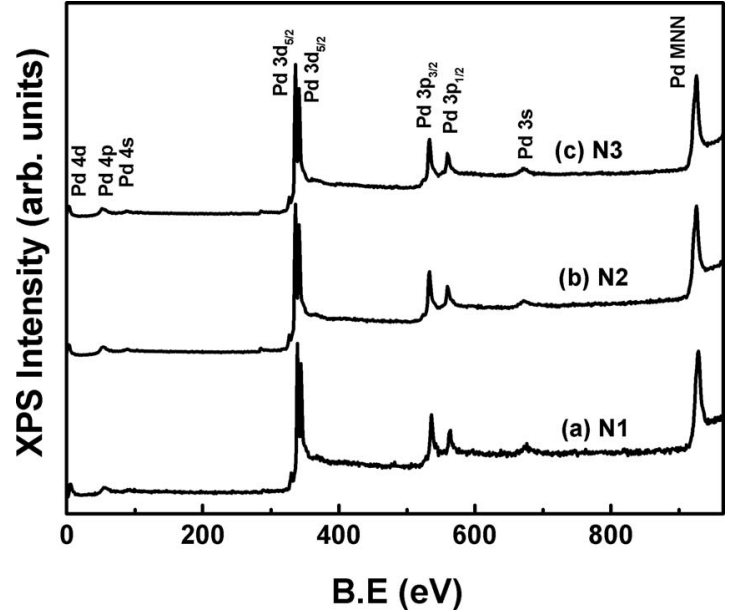

FIG. 3. XPS survey spectra of sputter cleaned Pd samples (a) N1, (b) N2, and (c) N3

that the binding energy of $\mathrm{Pd} 3 d$ spectra in nanoparticle samples N1, N2, and N3 has been observed to be shifted, respectively, by $0.6,0.3$, and $0.1 \mathrm{eV}$ as compared to bulk Pd. The centroid of the $4 d$ XPS valence band spectrum of the nanoparticle samples has been observed to shift to higher energy side by $0.7,0.6$, and $0.4 \mathrm{eV}$, respectively, as shown in Fig. 4(b). A similar positive shift in the binding energy in core and valence electrons with decrease in the particle size has been observed in many metals $(\mathrm{Au}, \mathrm{Ag}, \mathrm{Pd}, \mathrm{Ni}$, and $\mathrm{Cu})$ at nanodimensions. For example, a shift of $0.4 \mathrm{eV}$ in the $3 d$ core and $0.6 \mathrm{eV}$ in the valence electrons for $5 \mathrm{~nm} \mathrm{Ag}$ nanoparticles and a shift of $1.0 \mathrm{eV}$ in the $4 d$ centroid and $1.3 \mathrm{eV}$ in the core electrons for $1 \mathrm{~nm} \mathrm{Pd}$ nanoparticles have been reported in literature. ${ }^{3,5,7}$
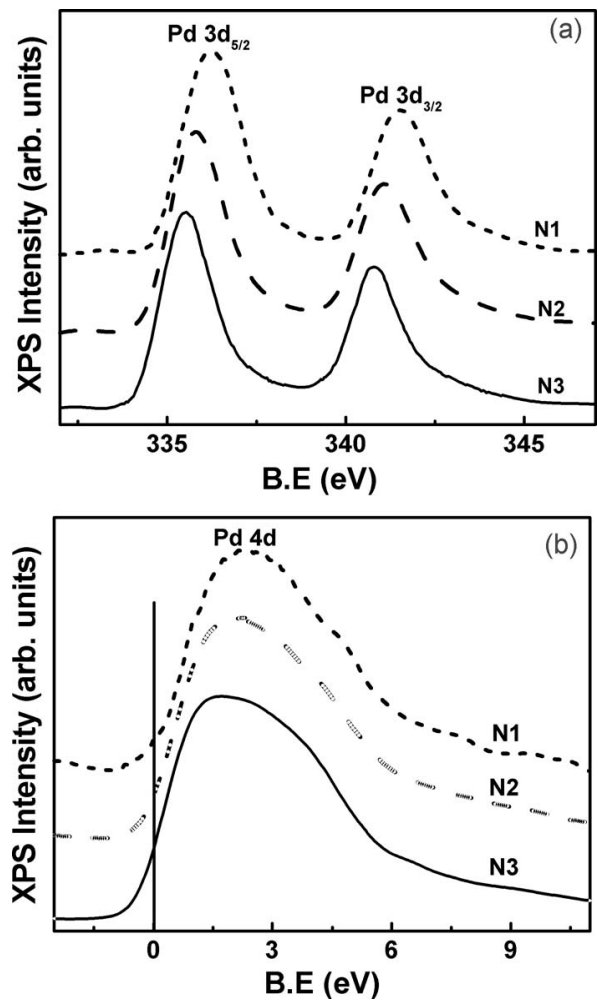

FIG. 4. (a) Pd $3 d$ core level and (b) Pd $4 d$ valence band spectra of sputter cleaned samples N1, N2, and N3. 


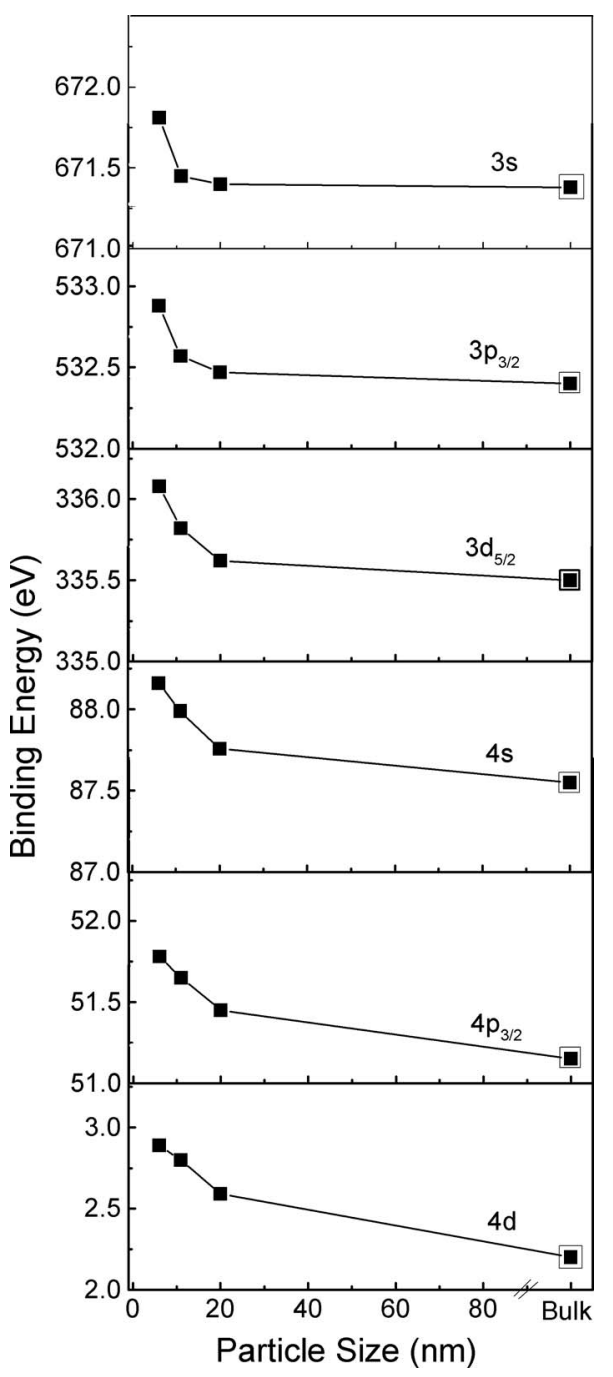

FIG. 5. Variation in the binding energy of various valence and core electrons in $\mathrm{Pd}$ as a function of nanoparticle size. For comparison, the corresponding binding energies in bulk Pd are also plotted (回)

It is interesting to note that the shift in the binding energy of Pd $4 d$ valence electrons has been observed to be more than that of the Pd $3 d$ core electrons. This observation indicates the effect of the nanoparticle nature influence on the valence and core electrons differently. To understand this phenomenon in detail, XPS spectra of all the core and valence electrons were recorded. The binding energy of the various core and valence electrons in the Pd nanoparticle samples N1, N2, and N3 and the bulk Pd is shown in Fig. 5. It is evident that there is a sharp variation in the binding energy of the inner core levels as compared to the outer core and valence electrons. Figure 6 shows a log-log plot of the shift in the binding energy $(\Delta \mathrm{BE})$ of the various core and valence electrons as a function of particle size $(R)$. The size dependence of the binding energy shift of various electrons is clearly different. It is interesting to note that the dependence of binding energy shift on particle size (the slope $x$ of the log-log plots given in Fig. 6) is more (2.5) for inner core level electrons as compared to the valence electrons $(0.5)$. From this one expects that with the decrease in the particle size, the core level shift should become more than the valence level shift. However, for the Pd nanoparticles reported

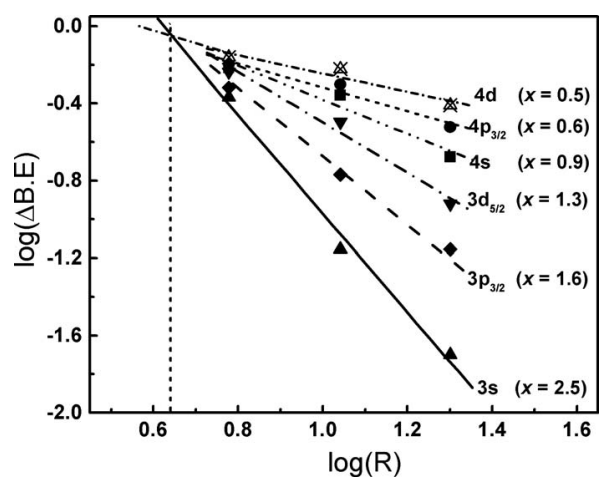

FIG. 6. The log-log plot of the shift in the binding energy $(\Delta \mathrm{BE})$ as a function of nanoparticle size $(R)$ of the various Pd valence and core electrons. The values of the corresponding negative slopes are also mentioned.

in the present study (e.g., for Pd nanoparticle of size $11 \mathrm{~nm}$ ), it is observed that the amount of shift in the binding energy of valence electrons $(0.6 \mathrm{eV})$ is more compared to the inner core electrons $(0.3 \mathrm{eV})$. This interesting behavior can be understood in terms of the interplay between the quantum size effects and coordination reduction. As revealed from the GAXRD measurements, lattice strain increases with decrease in the Pd nanoparticle size. In addition, the changes in the electronic structure of nanoparticle due to confinement effects are inevitable. The quantum confinement effect as well as the lattice strain, and hence the coordination number reduction, is reported to induce the binding energy shifts. ${ }^{1,9}$ It is interesting to note that the effects due to quantum confinement have larger dependence on particle size $\left(\sim R^{-2}\right)$ than those due to coordination reduction $\left(\sim R^{-1}\right){ }^{17,18}$ Indicating that the inner core electrons are more strongly affected by the quantum confinement, the valence electrons are strongly affected by the coordination reduction. Thus as the nanoparticle size decreases, the interplay between the quantum confinement and coordination reduction results in varied shifts in various electronic levels, and consequently, the inner core level shifts become more than the valence level shifts at a very small nanoparticle size. Although the Pd nanoparticles studied in this report are far from this size regime, it is interesting to note that on extrapolation of the log-log plots (dotted lines in Fig. 6) to smaller nanoparticle size, a critical particle size below which the binding energy shift of the inner core electrons is more than the valence electrons can be obtained. This reveals that the core electron effects are due to quantum confinement. This is in good agreement with the binding energy shifts reported in literature for the Pd nanoparticles (Fig. 7). ${ }^{1,3,5}$

In addition to the positive shift in the binding energy, a change in the FWHM of the Pd valence and the core levels is observed. The $4 d$ valence band and the outer core levels $4 p$ and $4 s$ show a decrease, whereas the $3 s, 3 p$, and $3 d$ inner core levels exhibit an increase in the FWHM, revealing a different origin of the effect responsible for the varied behavior. The narrowing of the valence $d$ band with decreasing nanoparticle size has been observed in XPS studies of Au, $\mathrm{Ag}$, and $\mathrm{Pd}$ and has been attributed to the reduced coordination with decrease in nanoparticle size. ${ }^{3,7,8}$ These line widths include the width of the $M g K \alpha$ line and other instrumental 


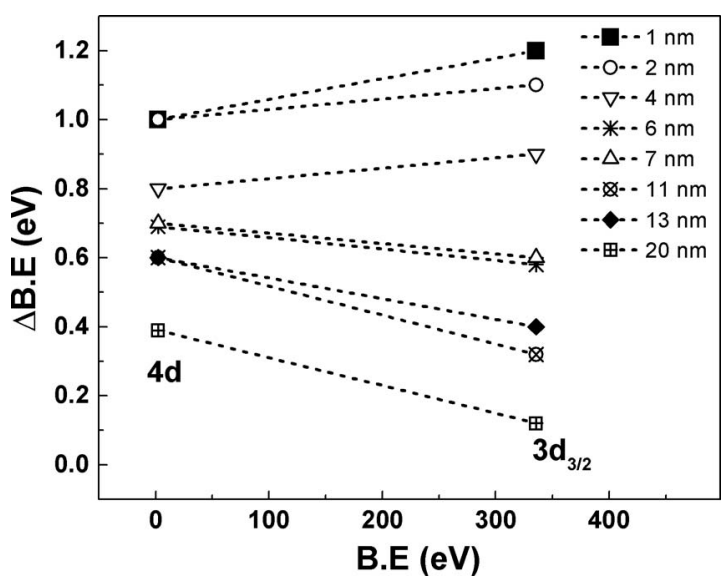

FIG. 7. The shift in the binding energy of the $4 d$ valence and $3 d_{5 / 2}$ core electrons in Pd nanoparticle samples N1 $(6 \mathrm{~nm}), \mathrm{N} 2(11 \mathrm{~nm})$, and N3 (20 $\mathrm{nm})$ with respect to bulk Pd. The corresponding shifts reported in literature (Refs. 1, 3, and 5) for Pd at nanodimension are also shown.

effects. The instrumental effects in the present study, however, cannot mask the substantial changes caused by the decrease in nanoparticle size. As compared to the spectrum of sample N3, the Pd $4 d$ valence band spectra in the case of nanoparticle samples $\mathrm{N} 1$ and $\mathrm{N} 2$ show a rounding off at the upper edge of the band [Fig. 4(b)]. The rounding off of the valence band spectra in the case of Pd and Pt at nanodimensions has been attributed to the loss of long-range periodicity. ${ }^{1}$ The weakening of the peak near $E_{F}$ does not imply a loss in occupied $d$ states but only a redistribution of states within the band. ${ }^{3}$ The increase in the FWHM in core level is attributed to the increase in the surface to bulk atom ratio, the presence of more localized unoccupied $d$ states at the Fermi level, ${ }^{8}$ and to the phonon excitations that contribute a Gaussian-type broadening to core line shapes. ${ }^{4}$

\section{CONCLUSION}

The present study gives a detailed analysis of modification in the electronic structure of Pd nanoparticles synthesized by inert gas evaporation technique in the size range of 6-20 $\mathrm{nm}$. The shift in the binding energy of Pd $4 d$ valence electrons is more than that of the $\mathrm{Pd} 3 d$ core electrons. The interplay between quantum size effects and coordination re- duction and the varied size dependence of the two effects $\left(\sim R^{-2}\right.$ and $R^{-1}$, respectively) explains these results. The FWHM of the $4 d, 4 p$, and $4 s$ peaks decreases, whereas that of $3 s, 3 p$, and $3 d$ peaks increases with decrease in size, pointing toward the difference in the origin of the effects responsible for modifying the core and valence electron levels with reduction in size.

\section{ACKNOWLEDGMENTS}

One of the authors (I.A.) is grateful to the University Grants Commission (UGC), India for providing the Senior Research Fellowship (SRF) in carrying out this work. This work has been carried out as a part of the project (Grant No. SR/S5/NM-90/2006) sponsored by the Nano Mission of Department of Science and Technology, Government of India.

${ }^{1}$ M. G. Mason, Phys. Rev. B 27, 748 (1983).

${ }^{2}$ G. K. Wertheim, Z. Phys. B: Condens. Matter 66, 53 (1987); Z. Phys. D: At., Mol. Clusters 12, 319 (1989).

${ }^{3}$ G. K. Wertheim, S. B. DiCenzo, and D. N. E. Buchanan, Phys. Rev. B 33, 5384 (1986).

${ }^{4}$ B. Richter, H. Kuhlenbeck, H.-J. Freund, and P. S. Bagus, Phys. Rev. Lett. 93, 026805 (2004)

${ }^{5}$ H. N. Aiyer, V. Vijayakrishnan, G. N. Subbanna, and C. N. R. Rao, Surf. Sci. 313, 392 (1994).

${ }^{6}$ Y. Wu, E. Garfunkel, and T. E. Madey, J. Vac. Sci. Technol. A 14, 2554 (1996).

77. Pászti, G. Petö, Z. E. Horváth, A. Karacs, and L. Guczi, Solid State Commun. 107, 329 (1998)

${ }^{8}$ O. Cheshnovsky, K. J. Taylor, J. Conceicao, and R. E. Smalley, Phys. Rev. Lett. 64, 1785 (1990).

${ }^{9}$ B. Richter, H. Kuhlenbeck, H.-J. Freund, and P. S. Bagus, Phys. Rev. Lett. 93, 026805 (2004).

${ }^{10}$ I. Aruna, B. R. Mehta, and L. K. Malhotra, Appl. Phys. Lett. 87, 103101 (2005).

${ }^{11}$ G. K. Wertheim and P. H. Citrin, in Photoemission in Solids I, edited by M. Cardona and L. Ley (Springer, Berlin, 1978).

${ }^{12}$ D. Briggs and M. P. Seah, Practical Surface Analysis by Auger and X-ray Photoelectron Spectroscopy (Wiley, Chichester, UK, 1983).

${ }^{13}$ L. G. Berry, Powder Diffraction Files for Inorganic Materials (Joint Committee on Powder Diffraction Standards, Pennsylvania, 1972), p. 461043.

${ }^{14}$ Z. M. Stadnik, P. Griesbach, G. Dehe, P. Gütlich, T. Kohara, and G. Stroink, Phys. Rev. B 35, 6588 (1987).

${ }^{15}$ C. Q. Sun, J. Phys.: Condens. Matter 11, 4801 (1999).

${ }^{16}$ G. K. Williamson and W. H. Hall, Acta Metall. 1, 22 (1953).

${ }^{17}$ C. Q. Sun, T. P. Chen, B. K. Tay, S. Li, H. Huang, Y. B. Zhang, L. K. Pan, S. P. Lau, and X. W. Sun, J. Phys. D 34, 3470 (2001).

${ }^{18}$ C. Q. Sun, Phys. Rev. B 69, 045105 (2004) 